\title{
Surface potential of chalcopyrite films measured by KPFM
}

\author{
S. Sadewasser* \\ Hahn-Meitner Institut, Glienicker Str. 100, 14109 Berlin, Germany \\ Received zzz, revised zzz, accepted zzz \\ Published online $z z z$ \\ PACS 68.37.Ps, 68.47.Fg, 68.60.-p, 73.61.Ga, 73.20.At, 73.40.Cg
}

Atomic force microscopy is widely used to characterize the surface topography of a variety of samples. Kelvin probe force microscopy (KPFM) additionally allows determining images of the surface potential with nanometer resolution. The KPFM technique will be introduced and studies on surfaces of chalcopyrite semiconductors for solar cell absorbers will be presented. It is shown that operation in ultra-high vacuum (UHV) is required to obtain meaningful work function values. Different methods for obtaining UHV-clean surfaces are presented and KPFM studies on these are compared. Surfaces where prepared by in-vacuum deposition, inert-gas transfer, in-vacuum decapping of a protective Se-cap and a peel-off method. Finally, a sputter-annealing cycle also allows to obtain well-suited surfaces for KPFM studies. Employing KPFM, variations in the local surface potential at grain boundaries of polycrystalline $\mathrm{CuGaSe}_{2}$ films were observed. A potential drop indicates the presence of charged defects at grain boundaries. Furthermore, different electronic activity was found for different grain boundaries, as concluded from studies under illumination. Using laterally resolved surface photovoltage, $\mathrm{a} \mathrm{Cu}_{2-x} \mathrm{Se}$ impurity phase could be observed in $\mathrm{CuGaSe}_{2}$.

Copyright line will be provided by the publisher

1 Introduction Photovoltaic energy conversion is one route followed in the quest for a sustainable energy supply in the future. Thin film solar cell technologies offer a high cost reduction potential compared to standard silicon wafer technology. With their high power conversion efficiencies on the laboratory scale, thin film solar cells from the $\mathrm{Cu}$-chalcopyrite materials system are a promising candidate for this technology; in fact, pilot production lines have recently been started. Currently, the highest efficiency of nearly $20 \%$ is obtained for $\mathrm{Cu}(\mathrm{In}, \mathrm{Ga}) \mathrm{Se}_{2}$ containing $\approx 30 \%$ of $\mathrm{Ga}$ [1]. A typical solar cell consists of a metallic Mo layer on a float glass substrate, a polycrystalline p-type $\mathrm{Cu}$-chalcopyrite absorber layer with a typical thickness of $2 \mu m$, a thin buffer layer (typically $\mathrm{CdS}$ ) and the n-type double layer window $(\mathrm{ZnO})$. A NiAl grid provides the front contact. A wide variety of growth techniques for the chalcopyrite absorber have been studied, including evaporation, sputtering and subsequent rapid thermal processing in chalcogen atmosphere, chemical vapor deposition, metal organic vapor phase epitaxy and electrochemical deposition. In such multilayered structures the various interfaces play a crucial role, as they are prone to contain electronically active defects causing electronic losses in the device. The polycrystalline character of the materials introduces additional effects, as for example varying composition in different grains and grain boundaries [2]. Recently, also compositional variations on the nanometer scale have been proposed [3]. Therefore, studies of surfaces and interfaces are highly important in order to further the understanding of these materials and eventually increase their efficiency towards the theoretical limit of $\approx 30 \%$ [4].

Compared to Si or III-V semiconductors, reported surface studies on chalcopyrite semiconductors are rather limited. R. Scheer [5] has reviewed some early studies with a focus on surface conditioning by physical and chemical methods. Also some photoemission spectroscopy (PES) studies have been reported (see for example [6]). However, information about lateral variations on a length scale of the grain size is not available in these studies. With the development of atomic force microscopy (AFM) a widely applied tool

* Corresponding author: e-mail: sadewasser@hmi.de, Phone: +4930 8062 2164, Fax: +49 3080623199 
for surface characterization has become available [7]. The AFM has been extended by combination with various surface characterization techniques allowing access to a variety of information with high spatial resolution [8]. One representative of these enhanced AFM is the Kelvin probe force microscope (KPFM) which allows the spatially resolved measurement of the contact potential [9]. In recent years, KPFM has also been applied to the characterization of chalcopyrite solar cell materials and devices [10, 11]. This paper will introduce the KPFM technique and review a variety of KPFM studies on chalcopyrite materials. The surface condition is a crucial point in electronic surface characterization, therefore the paper will focus on studies performed under ultrahigh vacuum (UHV) conditions.

2 Experimental The KPFM is based on the non-contact AFM (NC-AFM), which measures the topography of a sample by means of the change of the cantilever's resonance frequency when tip-sample forces act on the cantilever [12]. In the frequency modulation technique (FM-mode), normally applied in UHV, the tip-sample distance is controlled by maintaining a constant frequency shift with respect to the free resonance frequency [13]. The cantilever is sensitive to the force gradient between tip and sample, thus providing for the high spatial resolution of NC-AFM [14]. The KPFM specifically makes use of the electrostatic forces between tip and sample and allows the measurement of the contact potential [9]. In addition to the normally applied dc voltage $\left(V_{d c}\right)$ between tip and sample, an ac-voltage $V_{a c} \sin (\omega t)$ at a frequency $\omega$ is applied. This results in an oscillating electrostatic force, which induces an oscillation of the cantilever at the frequency $\omega$. The tip-sample system can be considered as a capacitor, and the electrostatic force can be expressed as:

$$
F_{e l}=-\frac{1}{2} \frac{\partial C}{\partial z} V^{2}(t)=-\frac{1}{2} \frac{\partial C}{\partial z}\left[V_{d c}-V_{C P}+V_{a c} \sin (\omega t)\right]^{2} .
$$

where $\partial C / \partial z$ is the capacitance gradient of the tip-sample system and $V_{C P}=\Delta \Phi / e$ the contact potential, which is the difference in work function between tip and sample ( $e$ is the elementary charge). Equation (1) can be written as $F_{e l}=F_{d c}+F_{\omega}+F_{2 \omega}$, with the spectral components:

$$
\begin{aligned}
& F_{d c}=-\frac{\partial C}{\partial z}\left[\frac{1}{2}\left(V_{d c}-V_{C P}\right)^{2}+\frac{V_{a c}^{2}}{4}\right], \\
& F_{\omega}=-\frac{\partial C}{\partial z}\left(V_{d c}-V_{C P}\right) V_{a c} \sin (\omega t), \\
& F_{2 \omega}=\frac{\partial C}{\partial z} \frac{V_{a c}^{2}}{4} \cos (2 \omega t) .
\end{aligned}
$$

The dc part $F_{d c}$ contributes to the topography signal, $F_{\omega}$ at the ac-frequency is used to measure the contact potential and the contribution $F_{2 \omega}$ can be used for capacitance microscopy. Using a lock-in amplifier the amplitude of the cantilever oscillation at $\omega$ is detected. This signal is minimized by controlling $V_{d c}$ to match the contact potential $V_{C P}$, as can be seen from Eq. (3). Recording $V_{d c}$ while scanning the topography, an image of the contact potential is obtained. Using a tip with a known work function (e.g. by calibration against highly oriented pyrolytic graphite - HOPG) allows to derive the sample's work function [15].

The present set-up is a modified Omicron UHV-AFM/STM operating at a base pressure $<10^{-10} \mathrm{mbar}$ [15]. The amplitude modulation technique (AM mode) is used for the measurement of the contact potential; this mode detects the long range electrostatic force. The ac-frequency $\omega$ is tuned to the second resonance frequency of the cantilever [16]; this resonance-enhanced technique provides an improved sensitivity and allows the independent and simultaneous imaging of the topography and contact potential. We readily obtain an energy resolution of $5 \mathrm{meV}$ using ac-voltages as low as $100 \mathrm{mV}$. Large ac-voltages possibly induce band bending at the surface of semiconductors, which would cause an incorrect determination of the work function. Therefore, tuning a low ac-voltage to the second resonance mode is advantageous.

For semiconductors in general and for photovoltaic applications in specific, the effect of sample illumination presents an important source of information in the characterization of samples. Illumination with 
super band gap light will cause generation of electron-hole pairs which can be separated in internal fields of the semiconductor structure [17]. This surface photovoltage (SPV) can be spatially resolved with the KPFM. To visualize the contributing effects a general band diagram is shown in Fig. 1. A surface space charge region develops due to charges located at the surface, as depicted in Fig. 1 (a) for a semiconductor in the dark (solid line). The work function $\Phi$ is the energy difference between the Fermi level $\mathrm{E}_{F}$ and the local vacuum level $\mathrm{E}_{l v}$ at the surface. A possible surface dipole $\Delta \phi_{s}$ modifies the work function accordingly. Under illumination (dotted line) the band bending is modified; in the case of the presented p-type semiconductor $\Phi$ is increased. The magnitude of the surface dipole is not affected. In Fig. 1 (b) a possible contribution of interfaces is shown. Light-induced charge carrier generation will also cause band flattening at interfaces. When the sample is contacted via the burried layer, then the SPV will contain both contributions, from the interface and from the surface. If the illumination intensity is sufficient, flat band conditions at the surface can result [17]. A detailed discussion of SPV can be found in Ref. [17]. In the presented experiments, we used a red laser with $\lambda=675 \mathrm{~nm}(20 \mathrm{~mW}$ max. intensity).

\section{Results}

3.1 UHV-clean surfaces Whereas topography measurements by AFM are only negligibly affected by surface cleanness, the electronical information of the work function requires clean surfaces [15]. Therefore, surfaces to be meaningfully characterized by KPFM have to be either prepared or cleaned in the UHV. It has been shown, that $\mathrm{Cu}$-chalcopyrite surfaces oxidize when exposed to air [18]. In principle, the observation of laterally inhomogeneous samples is also possible on oxidized and adsorbate-covered surfaces, however, the absolute magnitude of contact potential differences within the surface has to be carefully discussed, as different regions of the sample could be affected to a different extent. In this section, different preparation techniques for obtaining a clean surface in UHV will be presented; the next section will discuss details of a sputter-annealing cleaning procedure in UHV.

The most straight forward method to obtain a UHV-clean sample surface is to grow the chalcopyrite thin film inside the same UHV chamber. For this purpose we have built up a UHV physical vapor deposition (PVD) system, which can be directly connected to the UHV-KPFM [20]. The elements $\mathrm{Cu}, \mathrm{Ga}$ and $\mathrm{Se}$ are thermally evaporated from effusion cells onto a heated substrate. A two-stage growth process was optimized for sample growth on a $1 \times 1 \mathrm{~cm}^{2}$ glass/Mo substrate. In the first growth step, a $\mathrm{Cu}$-rich film is grown to ensure good structural film properties. The second growth step consists of only $\mathrm{Ga}$ and $\mathrm{Se}$ and converts the $\mathrm{CuGaSe}_{2}$ into a Ga-rich film with good electronic properties and at the same time avoids segregated $\mathrm{Cu}_{2-x} \mathrm{Se}$ foreign phases [19]. Solar cells processed from these absorbers show efficiencies up to $5 \%$. The topography and work function of the as-prepared film are shown in Fig. 2 (a) and (b), respectively. The granular structure of the polycrystalline thin film with a typical grain size of $\approx 500 \mathrm{~nm}$ is seen. The work function shows variations within $250 \mathrm{meV}$; the distribution of work functions is best seen in a histogram as shown in Fig. 3 (a). A single peak in the distribution can well be fitted by a Gauss distribution with a maximum at $\Phi=5.45 \mathrm{eV}$ and a full width at half maximum (FWHM) of $\approx 0.06 \mathrm{eV}$.

For most investigations, samples from an in-vacuum deposition and transfer are not available. Therefore, other ways of obtaining clean as-grown surfaces in UHV are necessary. One option is transfer in an inert-gas atmosphere (i.e. Ar or $\mathrm{N}_{2}$ ), which avoids surface oxidation and adsorbate contamination [21]. As

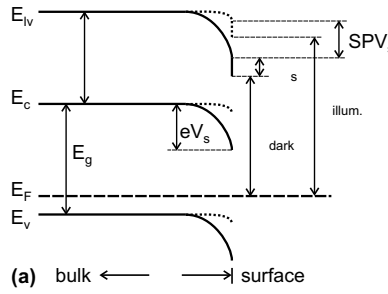

(b)

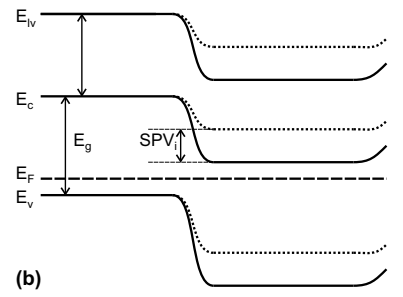

Fig. 1 Schematic band diagrams showing (a) the work function in the dark (solid lines) and under illumination (dotted lines), the reduction of surface band bending upon illumination and the possible presence of a surface dipole. (b) shows the contribution of interfaces and surfaces to the surface photovoltage. 


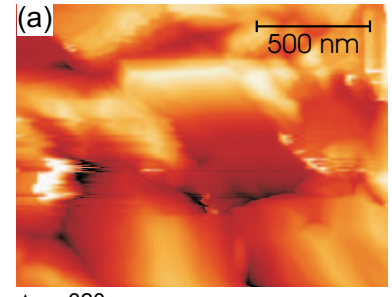

$\Delta \mathrm{z}=320 \mathrm{~nm}$

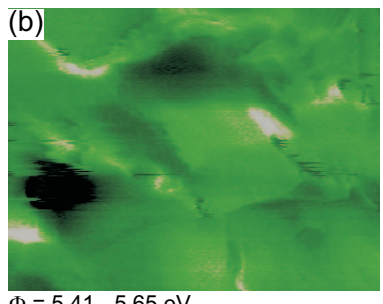

$\Phi=5.41-5.65 \mathrm{eV}$
Fig. 2 KPFM measurement of a $\mathrm{CuGaSe}_{2}$ thin film deposited in a UHV-PVD and transferred under UHV conditions. The topography (a) shows the granular structure $(\Delta \mathrm{z} \approx 320 \mathrm{~nm})$ and the work function (b) varies from $\Phi \approx 5.41$ to $5.65 \mathrm{eV}$. [20]

an example, a $\mathrm{CuGaSe}_{2}$ surface prepared on a Mo/glass substrate in a chemical vapor deposition (CVD) reactor is shown in Fig. 4 [10, 22]. The granular structure in the topography is comparable to the previous case; however, the work function shows a clear distinction between the values for different facets of the crystallites. This is also clearly observed in the corresponding histogram, presented in Fig. 3 (b). A number of different peaks can be observed, each corresponding to a distinct $\Phi$-value of a specific facet [10].

Another option for transferring a sample from the growth system to the UHV analysis chamber without oxidation and contamination is to overgrow the surface with a protective layer, which can be removed inside the UHV. A well-suited option for this overlayer is a Se-capping layer of a few hundred nanometers thickness, which is deposited after film growth by the same Se-source used for the chalcopyrite deposition. Such a film can be transported in air without oxidation of the burried chalcopyrite and the protective layer can be evaporated by sample heating inside the UHV chamber. Hunger et al. [23, 24] have shown by PES that the resulting chalcopyrite surface is free of oxides and other contamination and shows a composition similar to UHV-prepared samples. We have followed this route using a co-evaporated $\mathrm{Cu}(\mathrm{In}, \mathrm{Ga}) \mathrm{Se}_{2}$ thin film. Topography and work function images (not shown) obtained by KPFM show similar results to the previous samples [25] and the histogram of the work function image is presented in Fig. 3 (c).

Another, yet different approach for obtaining a clean surface in UHV is the preparation by peel-off $[21,26]$. In this technique, use is made of an intermediate van-der-Waals compound at the chalcopyrite/Mo
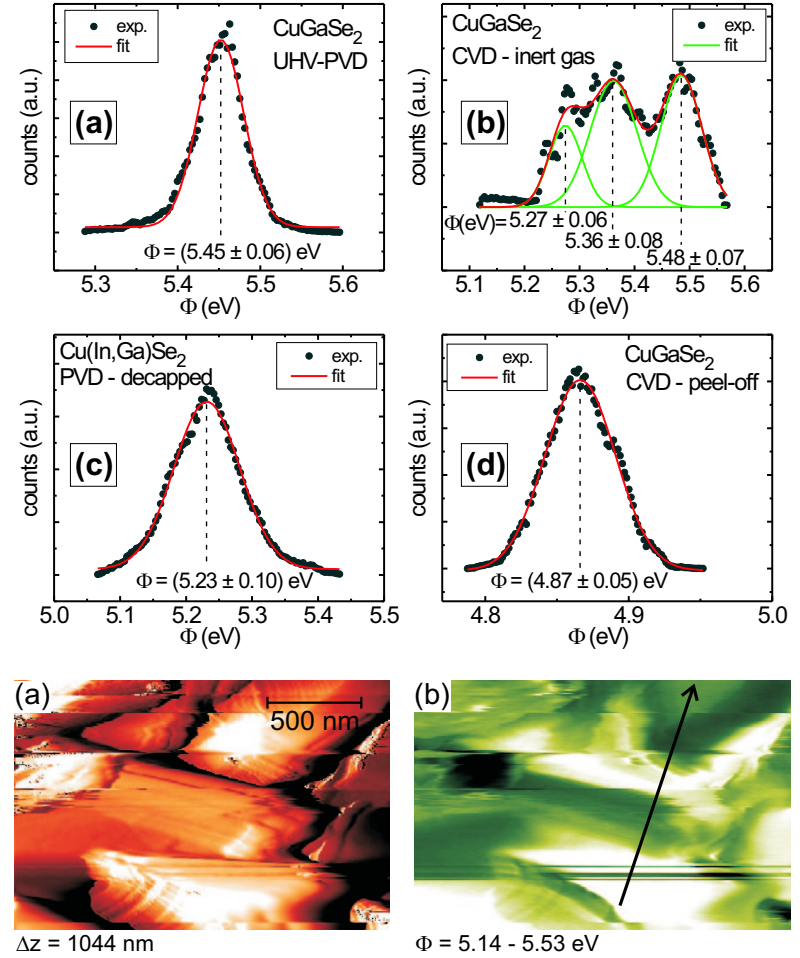



$\Phi=5.14-5.53 \mathrm{eV}$
Fig. 3 Histograms showing the work function distribution obtained by KPFM for (a) UHV-PVD grown $\mathrm{CuGaSe}_{2}$, (b) CVD-grown $\mathrm{CuGaSe}_{2}$ transferred in inert-gas atmosphere, (c) co-evaporated and $\mathrm{Se}$-decapped $\mathrm{Cu}(\mathrm{In}, \mathrm{Ga}) \mathrm{Se}_{2}$, (d) CVD-grown $\mathrm{CuGaSe}_{2}$ rear-side obtained by peel-off in UHV.

Fig. 4 KPFM measurement of a $\mathrm{CuGaSe}_{2}$ thin film deposited by CVD and transferred in inert-gas atmosphere (Ar). The topography (a) shows the granular structure $(\Delta \mathrm{z} \approx 1350 \mathrm{~nm})$ and the work function (b) varies from $\Phi \approx 5.14$ to $5.53 \mathrm{eV}$, showing distinct values for the different facets. [22] 

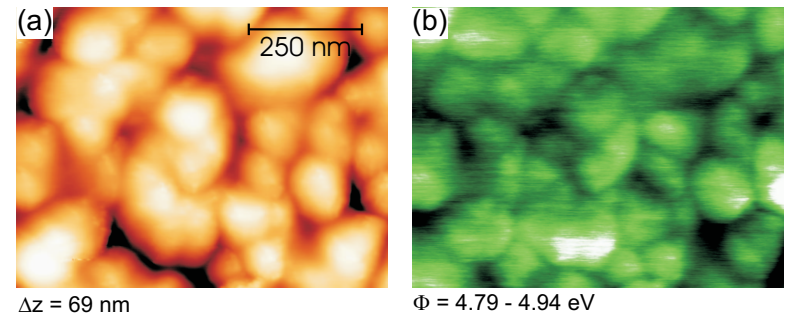

Fig. 5 KPFM measurement of the back-side surface of a $\mathrm{CuGaSe}_{2}$ thin film grown by CVD and obtained by peel-off in UHV. The topography (a) shows the granular structure $(\Delta \mathrm{z} \approx 69 \mathrm{~nm})$ and the work function (b) varies from $\Phi \approx 4.79$ to $4.94 \mathrm{eV}$. [27]

interface. During the deposition process, especially when CVD is used, a MoSe ${ }_{2}$ film develops on the surface of the Mo-layer. This layered compound shows weak bonding between van-der-Waals planes building up its crystalline structure. Gluing a supporting structure (i.e. a glass or metal foil) to the top of the chalcopyrite thin film, it can be lifted off from the Mo/glass substrate, provided it has the correct orientation of the $\mathrm{MoSe}_{2}$ layered structure with respect to the interface plane. The obtained rear-side chalcopyrite surface is free of Mo remnants, as confirmed by PES studies [21]. In Fig. 5 the topography (a) and work function (b) of this back-surface are shown [21, 27]. The granular structure can again be observed, however, the surface roughness is clearly lower and the grain size is considerably smaller. This is due to the initial growth, which starts from small grains which grow wider during the further growth of the thin film to its final thickness. In the work function image a fairly narrow range of values is observed as can be seen in the histogram in Fig. 3 (d); a lowering of $\Phi$ by $\approx 20$ to $50 \mathrm{mV}$ is observed at the grain boundaries [27]. It is apparent that the above observed work function variation according to distinct facets is not observed in the present case, pointing to a homogeneous texturing of the small initial grains.

The work function values obtained by Gauss-fitting the histograms in Fig. 3 are shown comparatively in Fig. 6 (a) for the differently prepared UHV-clean surfaces. A strict comparison is rather difficult and not very meaningful, as the growth processes are not identical and therefore also the materials composition and doping level is likely different; in the case of preparation by decapping, the In-containing $\mathrm{Cu}(\mathrm{In}, \mathrm{Ga}) \mathrm{Se}_{2}$ was used. Nevertheless, the obtained work function is fairly comparable for all samples showing values between $\approx 5.2 \mathrm{eV}$ and $5.6 \mathrm{eV}$. For the peeled-off $\mathrm{CuGaSe}_{2}$ a lower value is obtained. A PES study on this back-side surface revealed traces of oxygen and carbon, which was ascribed to contamination of the Mo substrate prior to the chalcopyrite deposition [21]; additionally also the Cu-rich initial growth of the 2-step grown $\mathrm{CuGaSe}_{2}$ might affect the work function on the rear-side. Also given in Fig. 6 (a) are the $\Phi$-values obtained under illumination (open symbols), from which the surface photovoltage can be computed, shown in the upper panel of Fig. 6 (a). The SPV shows a fairly constant value of $\approx 80 \mathrm{mV}$ for all surfaces.

3.2 Sputter-annealing cleaning procedure In many cases, samples can only be entered into the UHV after having been exposed to air. In this case a surface cleaning is necessary. This can well be performed by sputtering and annealing. Otte et al. [28] have performed PES studies on $\mathrm{CuInSe}_{2}$ and $\mathrm{CuGaSe}_{2}$ showing that soft sputtering with low energies up to 60 min results in cleaning from surface oxides and contamination, leading to a surface stoichiometry close to $1: 1: 2$. We have performed a study with subsequent sputter and annealing cycles in order to clean the surface of a $\mathrm{CuGaSe}_{2}$ sample grown in a PVD system [29] and exposed to air prior to introduction into the UHV-KPFM [30]. Fig. 7 (a) presents the topography image measured with KPFM showing the granular structure of the polycrystalline thin film. The simultaneously measured work function image for dark conditions is shown in Fig. 7 (b). Subsequent to this initial measurement the sample was cleaned by first annealing it at $170^{\circ} \mathrm{C}$ for $1 \mathrm{~h}$, then sputtering the surface with Ar-ions at $500 \mathrm{keV}$ for $90 \mathrm{~s}$ (incident angle varied between $45^{\circ}$ and $90^{\circ}$ to the surface), again annealing $\left(180{ }^{\circ} \mathrm{C}\right.$ for $\left.30 \mathrm{~min}\right)$, another sputtering step $(500 \mathrm{eV}$ for $10 \mathrm{~min})$ and a final annealing step at $180{ }^{\circ} \mathrm{C}$ for $30 \mathrm{~min}$. After each treatment the sample surface was imaged by KPFM. Measurements were performed in dark and under illumination. An overview of the obtained work function values is presented in Fig. 6 (b); the given $\Phi$-values correspond to the Gauss peak position and the error bar to the FWHM of the Gauss 


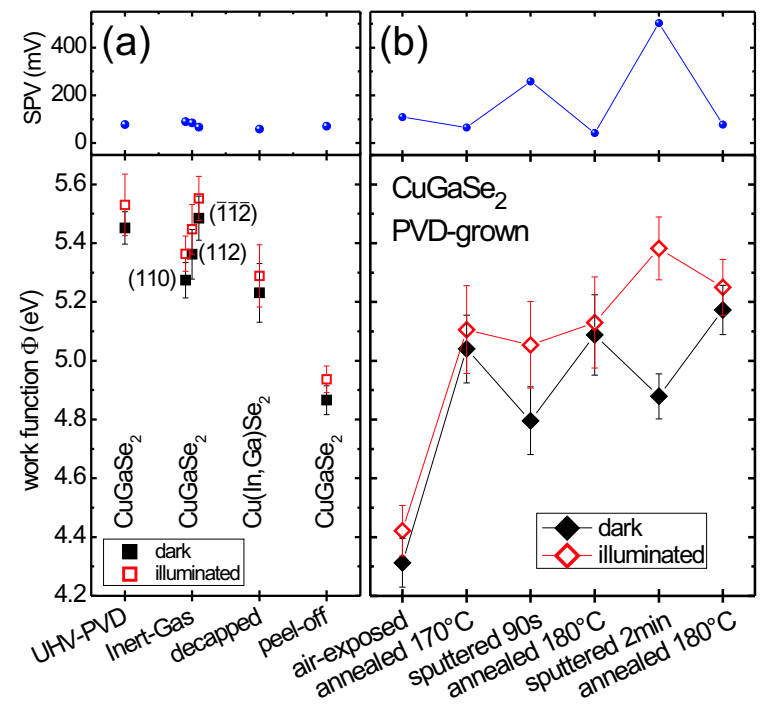

Fig. 6 Overview of the work function of (a) differently prepared UHV-clean surfaces of various chalcopyrite thin films (as indicated in the figure). The surface orientations of the $\mathrm{CuGaSe}_{2}$ after inert-gas transfer are taken from Ref. [10]. (b) Sputter and annealing cleaning procedure for an air-exposed PVDgrown $\mathrm{CuGaSe}_{2}$ thin film. The lower panels show the work function in dark and under illumination and the upper panels show the surface photovoltage (SPV).

distribution fitted to the corresponding work function histograms, according to the procedure shown in Fig. 3.

The initially low work function of $\approx 4.31 \mathrm{eV}$ is increased to $\approx 5.00 \mathrm{eV}$ after removal of the contamination water layer by the first annealing treatment. Subsequent sputtering lowers the work function slightly, despite the further cleaning, which is expected, i.e. the removal of oxides on the surface. A high SPV of $\approx 260 \mathrm{mV}$ is observed for the sputtered sample, indicating the presence of electrically active surface defects, presumably created due to the Ar-ion impact. Under illumination this sample reaches nearly the same work function as the annealed sample, indicating that band bending is efficiently reduced. The subsequent annealing treatment increases the work function and only a small SPV is observed. Upon annealing, surface atoms become more mobile resulting in defect healing. This reduces the band bending and accordingly also the SPV. Subsequent sputtering and annealing steps change the work function slightly, showing again the sputter-induced defects and high SPV. These results show that with sputter-annealing cycles an effective surface cleaning is obtained, at least from the electronic point of view. For testing the chemical composition PES studies are necessary. Very recently, a study on epitaxial $\mathrm{CuGaSe}_{2}$ showed that upon sputtering and simultaneous annealing at $300^{\circ} \mathrm{C}$ a $(2 \times 4)$ reconstructed surface is obtained, free of contamination and with a composition close to a 1:1:2 surface stoichiometry [31].

3.3 Lateral variations in the work function The strength of KPFM is the capability to measure contact potential with a high spatial resolution, as demonstrated by imaging different materials [11], different doping in semiconductors [32] and localized charges [33]. Looking at the work function images in Figs. 2, 5 and 7 most of the contrast in the images stems from dark "rings" around regions of constant work function. Comparison with the corresponding topography images reveals that the constant regions are related to

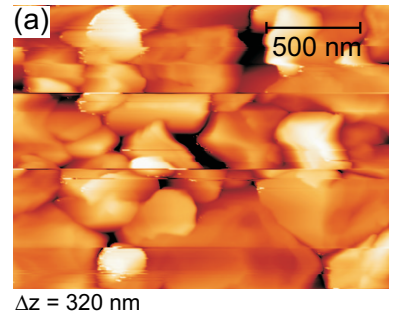

Fig. 7 KPFM measurement of PVD-grown $\mathrm{CuGaSe}_{2}$. The topography (a) shows the grains of the polycrystalline thin film $(\Delta \mathrm{z}=320 \mathrm{~nm})$ and (b) the simultaneously measured work function $(\Phi=$ $4.19-4.44 \mathrm{eV})$. 

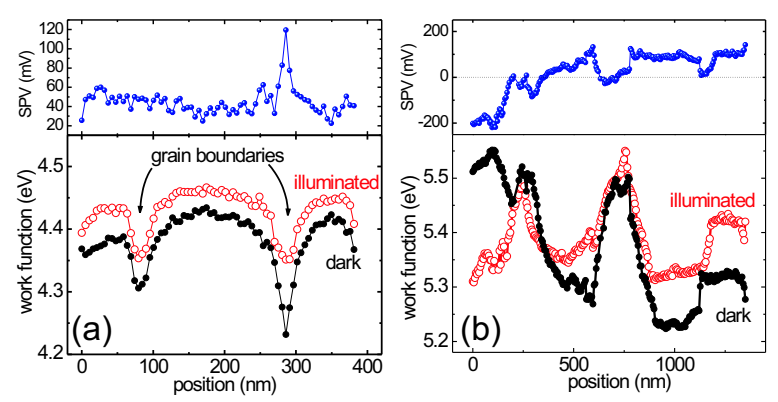

Fig. 8 Line profiles of the work function (lower panels) and surface photovoltage (upper panels) of (a) PVD-grown $\mathrm{CuGaSe}_{2}$ (see Fig. 7 (b)) showing a work function lowering at grain boundaries [34] and of (b) CVD-grown $\mathrm{CuGaSe}_{2}$ (see Fig. 4 (b)), where a grain likely covered with $\mathrm{Cu}_{2-x} \mathrm{Se}$ can be identified by its negative SPV [22].

the grains and the lower work function surrounding the grains corresponds to the grain boundaries. In the histograms in Fig. 3 this lower work function of the grain boundaries cannot be directly observed, as the covered area in the images is too small. For closer analysis, a line profile across three grains comprising two grain boundaries of the PVD-grown $\mathrm{CuGaSe}_{2}$ thin film (see arrow in Fig. 7 (b)) is shown in Fig. 8 (a). A work function drop at the grain boundaries is clearly observed. This drop has been previously attributed to the presence of charged defects. Using Poisson's equation, the magnitude of the work function drop and the width of the space charge regions extending into the $\mathrm{CuGaSe}_{2}$ semiconductor can be used to calculate the doping concentration $p$ of the material and give an estimate of the density of charged defects at the grain boundary $P_{g b}$ [34]. In this case we found: $p=9 \times 10^{16} \mathrm{~cm}^{-3}$ and $P_{g b}=8 \times 10^{11} \mathrm{~cm}^{-2}$, respectively, in agreement with Hall-effect measurements performed on the same sample [19,34]. Looking at the SPV at the grain boundaries (upper panel in Fig. 8), it is observed that the left grain boundary shows the same SPV as the grains (SPV $\approx 40 \mathrm{mV}$ ), whereas the right grain boundary exhibits a much larger value of $120 \mathrm{mV}$. This indicates that the electrical activity is related to the specifics of the grain boundary [27]; for example, the orientation and the detailed atomic structure of the grain boundary likely play an important role [35]. This topic will be discussed in more detail elsewhere [36].

Figure 8 (b) shows a line profile along the line shown in Fig. 4 (b). The work function shows various different values for different facets, as was already seen by the several peaks in the corresponding histogram in Fig. 3 (b). The profile under illumination (open symbols) shows an increase of $\Phi$ in most positions (positive SPV), however, just on the left end of the line, a lowering of $\Phi$ under illumination is observed. This becomes more clear in the SPV profile given in the upper panel, which is positive except for the left side, where it shows $\mathrm{SPV} \approx-200 \mathrm{mV}$ [22]. In a previous study [37] on epitaxial $\mathrm{CuGaSe}_{2}$ grown on GaAs, a similar SPV of $\approx-300 \mathrm{mV}$ has been observed. After $\mathrm{KCN}$ etching, which removes $\mathrm{Cu}_{2-x} \mathrm{Se}$ surface phases, no SPV could be observed. Therefore, we interpret the present case as a $\mathrm{Cu}_{2-x} \mathrm{Se}$ layer covering $\mathrm{a} \mathrm{CuGaSe}_{2}$ grain. Thus, the observed SPV originates from the $\mathrm{CuGaSe}_{2}$ surface where it is positive, but a contribution from the $\mathrm{CuGaSe}_{2} / \mathrm{Cu}_{2-x}$ Se interface makes the SPV strongly negative. As the substrate is a p-type $\mathrm{CuGaSe}_{2}$, it can be concluded that this surface layer must be a $\mathrm{p}^{+}$-type $\mathrm{Cu}_{2-x} \mathrm{Se}$ [37].

4 Conclusion It was shown that UHV-KPFM represents a powerful tool for simultaneous structural and electronic surface characterization of semiconductors. For $\mathrm{Cu}$-chalcopyrite thin films different preparation methods for obtaining clean surfaces inside UHV were discussed. A sputter-annealing treatment was presented, which provides the possibility to obtain comparable results in KPFM studies. It was demonstrated that laterally resolved SPV is a valuable source of information, for example for identifying differently active grain boundaries or foreign phases in chalcopyrite thin films.

Acknowledgements The author is grateful to D. Fuertes Marrón, Th. Glatzel, K. Ishii, Ch. Kaufmann, A. Neisser, M. Rusu, S. Schuler, S. Siebentritt, and M.Ch. Lux-Steiner. 


\section{References}

[1] M.A. Contreras, K. Ramanathan, J. AbuShama, F. Hasoon, D.L. Young, B. Egaas, and R. Noufi, Prog. Photovolt: Res. Appl. 13, 209 (2005).

[2] S. Siebentritt and U. Rau, (Eds.), Wide-Gap Chalcopyrites (Springer Verlag, Berlin, 2006).

[3] Y. Yan, R. Noufi, K.M. Jones, K. Ramanathan, M.M. Al-Jassim, and B.J. Stanbery, Appl. Phys. Lett. 87, 121904 (2005).

[4] W. Shockley and H.J. Queisser, J. Appl. Phys. 32, 510 (1961).

[5] R. Scheer, Trends Vac. Sci. Technol. 2, 77 (1997).

[6] D. Schmid, M. Ruckh, and H.W. Schock, Appl. Surf. Sci. 103, 409 (1996).

[7] G. Binnig, C.F. Quate, and Ch. Gerber, Phys. Rev. Lett. 56, 930 (1986).

[8] P. De Wolf, R. Stephenson, T. Trenkler, T. Clarysse, T. Hanschel, and W. Vandervorst, J. Vac. Sci. Technol. B 18, 361 (2000).

[9] J.M.R. Weaver and D.W. Abraham, J.Vac. Sci. Technol. B 9, 1559 (1991); M. Nonnenmacher, M.P. OBoyle, and H.K. Wickramasinghe, Appl. Phys. Lett. 58, 2921 (1991).

[10] S. Sadewasser, Th. Glatzel, M. Rusu, A. Jäger-Waldau, and M.Ch. Lux-Steiner, Appl. Phys. Lett. 80, 2979 (2002).

[11] Th. Glatzel, D. Fuertes Marrón, Th. Schedel-Niedrig, S. Sadewasser, and M.Ch. Lux-Steiner, Appl. Phys. Lett. 81, 2017 (2002).

[12] Y. Martin, C.C. Williams, and H.K. Wickramasinghe, J. Appl. Phys. 61, 4723 (1987).

[13] T.R. Albrecht, P. Grütter, D. Horne, and D. Rugar, J. Appl. Phys. 69, 668 (1991).

[14] R. Wiesendanger, Scanning Probe Microscopy and Spectroscopy: Methods and Applications (Cambridge Univ. Press, Cambridge, 1994).

[15] Ch. Sommerhalter, Th.W. Matthes, Th. Glatzel, A. Jäger-Waldau, and M.Ch. Lux Steiner, Appl. Phys. Lett. 75, 286 (1999).

[16] A. Kikukawa, S. Hosaka, and R. Imura, Rev. Sci. Instrum. 67, 1463 (1996).

[17] L. Kronik and Y. Shapira, Surf. Sci. Rep. 37, 1 (1999).

[18] R. Würz, A. Meeder, D. Fuertes Marrón, Th. Schedel-Niedrig, A. Knop-Gericke, and K. Lips, Phys. Rev. B 70, 205321 (2004).

[19] S. Schuler, S. Nishiwaki, J. Beckmann, N. Rega, S. Brehme, S. Siebentritt, M.Ch. Lux-Steiner, Proceedings of the IEEE Conference, New Orleans, 2002.

[20] S. Sadewasser, K. Ishii, Th. Glatzel, M.Ch. Lux-Steiner, in: Polycrystalline Semiconductors VII- Bulk Materials, Thin Films, and Devices, eds. T. Sameshima, T. Fuyuki, H.P. Strunk, J.H. Werner (Scitech Publ., Uettikon am See, Switzerland, 2003), page 319.

[21] D. Fuertes Marrón, A. Meeder, S. Sadewasser, R. Würz, C.A. Kaufmann, Th. Glatzel, Th. Schedel-Niedrig, and M.Ch. Lux-Steiner, J. Appl. Phys. 97, 094915 (2005).

[22] S. Sadewasser, Th. Glatzel, M. Rusu, A. Meeder, D. Fuertes Marrón, A. Jäger-Waldau, M.Ch. Lux-Steiner, Mater. Res. Soc. Symp. Proc. 668, H5.4.1 (2001).

[23] R. Hunger, T. Schulmeyer, A. Klein, W. Jaegermann, K. Sakurai, A. Yamada, P. Fons, K. Matsubara, and S. Niki, Surf. Sci. 557, 263 (2004).

[24] T. Schulmeyer, R. Kniese, R. Hunger, W. Jaegermann, M. Powalla, and A. Klein, Thin Solid Films 451-452, 420 (2004).

[25] M. Rusu, Th. Glatzel, A. Neisser, C.A. Kaufmann, S. Sadewasser, and M.Ch. Lux-Steiner, submitted to Appl. Phys. Lett. (2006).

[26] R. Würz, D. Fuertes Marrón, A. Meeder, A. Rumberg, S.M. Babu, Th. Schedel-Niedrig, U. Bloeck, P. SchubertBischoff and M.Ch. Lux-Steiner, Thin Solid Films 431-432, 398 (2003).

[27] D. Fuertes Marrón, S. Sadewasser, Th. Glatzel, A. Meeder, and M.Ch. Lux-Steiner, Phys. Rev. B 71, 033306 (2005).

[28] K. Otte, G. Lippold, D. Hirsch, A. Schindler, and F. Bigl, Thin Solid Films 361-362, 498 (2000).

[29] S. Schuler, S. Nishiwaki, M. Dziedzina, R. Klenk, S. Siebentritt, and M.Ch. Lux-Steiner, Mat. Res. Soc. Symp. Proc. 668, H5.14.1 (2001).

[30] S. Sadewasser, in: Wide-Gap Chalcopyrites, Eds. S. Siebentritt and U. Rau (Springer Verlag, Berlin, 2006).

[31] Th. Deniozou, N. Esser, S. Siebentritt, Surf. Sci. 579, 100 (2005).

[32] M. Tanimoto and O. Vatel, J. Vac. Sci. Technol. B 14, 1547 (1995).

[33] Y. Rosenwaks, R. Shikler, Th. Glatzel and S. Sadewasser, Phys. Rev. B 70, 085320 (2004).

[34] S. Sadewasser, Th. Glatzel, S. Schuler, S. Nishiwaki, R. Kaigawa, M.Ch. Lux-Steiner, Thin Solid Films 431-432, 257 (2003).

[35] G. Hanna, Th. Glatzel, S. Sadewasser, N. Ott, H.P. Strunk, U. Rau, and J.H. Werner, Appl. Phys. A 82, 1 (2006).

[36] S. Sadewasser, in preparation.

[37] Ch. Sommerhalter, S. Sadewasser, Th. Glatzel, Th.W. Matthes, A. Jäger-Waldau, and M.Ch. Lux Steiner, Surf. Sci 482-485, 1362 (2001). 